\title{
Paper Title Different Duty Cycle Ratio and Brightness Of Visual Stimuli Change To Steady State Visual Evoked Potential Response
}

\author{
Zeki ORALHAN*¹, Mahmut TOKMAKÇI ${ }^{1}$
}

\author{
Accepted $3^{\text {rd }}$ September 2016
}

\begin{abstract}
Stimuli types are very crucial for the performance of electroencephalogram (EEG) based brain computer interface (BCI) systems. This study aims to investigate methods for obtaining higher information transfer rate (ITR) through duty cycle and brightness variation of visual stimuli which have high frequency for steady state visual evoked potential-based BCI. Although previous studies were concentrated on either duty cycle or brightness of stimuli separately, our study focused on the change of duty cycle ratio and brightness of stimuli at the same time. Duty cycle values of $40 \%, 50 \%$, and $60 \%$ were used. During the experiment, 16 flickering stimuli were used on liquid crystal display. Participants gazed to the flicker which had frequency of $15 \mathrm{~Hz}$. Canonical correlation analyses (CCA) was used for channel selection and frequency detection. According to the CCA, the maximum average accuracy of the experiment was $92.54 \%$ when the frequency of flicker was in beta band and its duty cycle was $40 \%$ with a brightness tuning wave. Under the same conditions stated above, average ITR was improved $16.1 \%$ according to the most commonly used flicker model which is square wave and has $50 \%$ duty cycle.
\end{abstract}

Keywords: Brain Computer Interface, EEG, Human-Computer Interaction and Systems, Steady State Visual Evoked Potential.

\section{Introduction}

Brain computer interface systems gives chance users to communicate with external devices without using peripheral neuromuscular systems. There are many brain imaging methods, such as electroencephalography (EEG), functional magnetic resonance imaging(fMRI), magnetoencephalography (MEG), and and near-IR spectroscopy (NIRS) have been adopted BCI systems [1], [2]. In BCI systems, the commands are identified by extracting Electroencephalograms (EEG) which measures the electric field generated from brain. EEG based BCI systems is preferred for other BCI system because of practical use, low cost, good response, portability [3].

There are some most common approaches to EEG based BCI systems that are P300 potentials, event-related (de)synchronization (ERD/ERS), slow cortical potentials, oscillatory activity, and visual evoked potentials (VEPs) [4-7].

The Steady state visual evoked potential (SSVEP) is a brain response modulated by the frequency of repetitive visual stimulus [8]. SSVEP is known to be most prominent at the occipital location over the visual cortex.

The SSVEP-based BCI has been preferred due to high signal to noise ratio and high information transfer rate (ITR) according to other BCI approaches [9]. Moreover, the SSVEP has a short training and response time [10].

The term of Information transfer rate is a very significant unit that has been used to evaluate overall performance of BCI systems [11]. Types of stimulus have very important role for high SSVEP response that related on ITR that can be described as

\footnotetext{
${ }^{1}$ Turk Telekomünikasyon A.Ş. 38104, Kayseri/Turkey

* Corresponding Author: Email: zeki.oralhan@turktelekom.com.tr Note: This paper has been presented at the $3^{\text {rd }}$ International Conference on Advanced Technology \& Sciences (ICAT'16) held in Konya (Turkey), September 01-03, 2016.
}

information transmitted in bits per minute (bpm).

Thus, there are many studies for investigate best stimuli type for SSVEP based BCI systems. Wu (2010) [12] proposed that \%40 duty cycle ratio when the flicker was $10 \mathrm{~Hz}$, SSVEP response reached highest value. Shyu et al. (2013) [13] made a SSVEPbased BCI system that using FPGA, which based on duty cycle ratio. Manyakov et al. (2013) [14] showed that sampled sinusoidal stimulation enabled for encoding more targets and elicited SSVEP response with a significantly better phase stability. Moreover, Chen et al. (2014) [15] presented sampled sinusoidal stimuli for SSVEP response. According to result of this study, ITR value was reached the highest one when the flicker was at low and high frequencies.

In recent years, researchers presented many approaches to make and develop SSVEP based BCI systems.

In this study we aimed to investigate effect the change of duty cycle ratio and brightness of stimuli at the same time, although previous studies were concentrated on either duty cycle or brightness of stimuli separately.

\section{Material and Method}

Five healthy participants (males, mean age 29 years, corrected to normal vision) joined experiments in this study. Participants didn't have any history of epilepsy and psychiatric disorders.

\subsection{Experimental Structure}

In the experiments, SSVEP stimulator software was created by C\# programming language. This SSVEP stimulator software is portable and functional. As shown in Figure 1, according to the main menu of software, colour of flickers and background, size of flickers, frequencies of flickers, quantity of flickers, brightness of flickers, duty cycle ratio of flickers could be changed by users. 


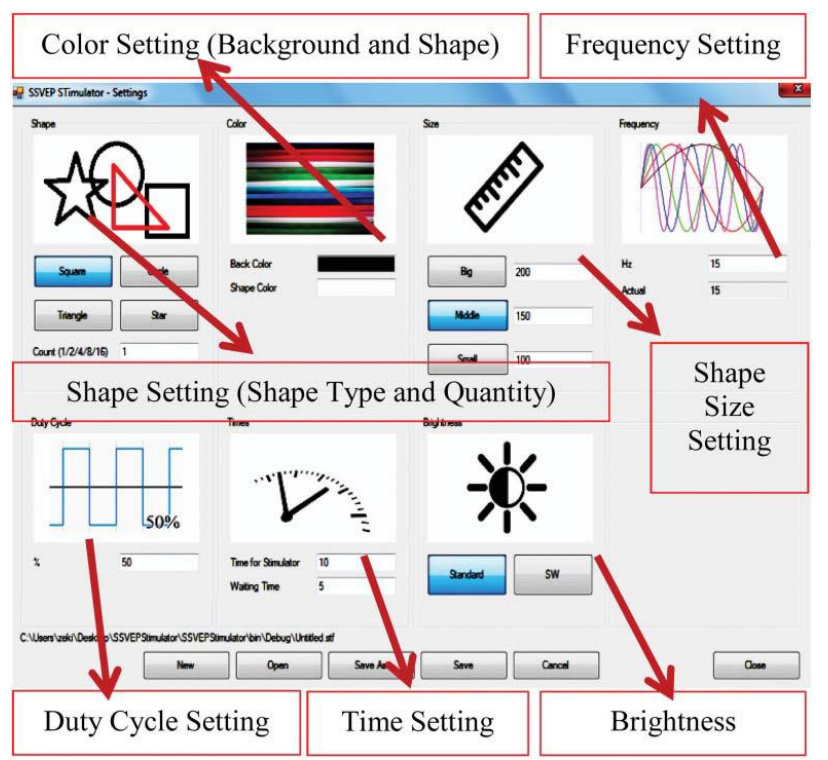

Figure 1. The SSVEP stimulator software setting menu.

The EEG were acquired from the scalp of the participants via 19 $\mathrm{Ag} / \mathrm{Ag}-\mathrm{Cl}$ electrodes. EEG electrodes placed according to the international 10-20 system. During the study Nihon Kohden Neurofax EEG-1200 EEG device was used for EEG data acquiring.

The reference electrodes were placed over the left and right earlobes. Ground electrode was on the intercilium point (the space between the eyebrows). EEG data were sampled at $500 \mathrm{~Hz}$.

\subsection{Stimulation Types}

In this study, we focused on the change of duty cycle ratio and brightness of stimuli at the same time. Duty cycle values of $40 \%$, $50 \%$, and $60 \%$ were used. As shown in Figure 2, we used a square pulse that was presented as Type I signal. $T_{\text {perioad }}$ is denoted the pulse cycle duration which is total of $T_{o n}$ and $T_{o f f}$ that are defined stimuli on and off cycle durations respectively. $R_{\text {duty }}$ is denoted duty cycle ratio which is presented in Equation 1.

$$
R_{\text {duty }}=T_{\text {on }}+T_{\text {off }}
$$

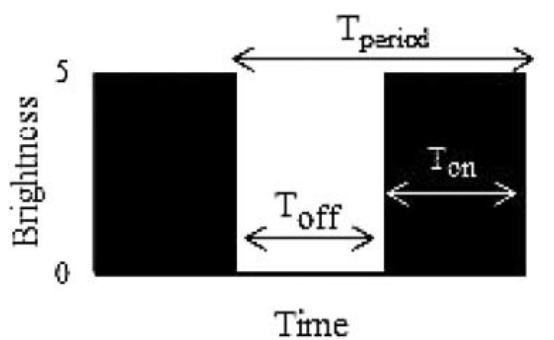

Figure 2. Type I signal: stimulation signal is at square pulse.

In this study, we presented a novel stimuli type that variation of brightness of pulse as shown in Figure 3. During the "on" status, flicker's brightness was on the increase within the equal time span of $T_{o n}=9$ till it reached to the peak value of Type I signal, after increased, it was on the decrease till it reached to "off" status within the equal time span.

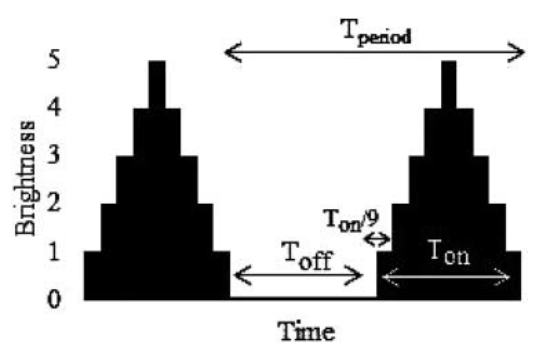

Figure 3. Type II signal: variable brightness when the flicker is on status

\subsection{Experimental Steps}

During this study, as shown in Figure 4, 16 flickering stimuli were showed on liquid crystal display (LCD). Participants were seated $50 \mathrm{~cm}$ away from LCD with a refresh rate of $60 \mathrm{~Hz}$.

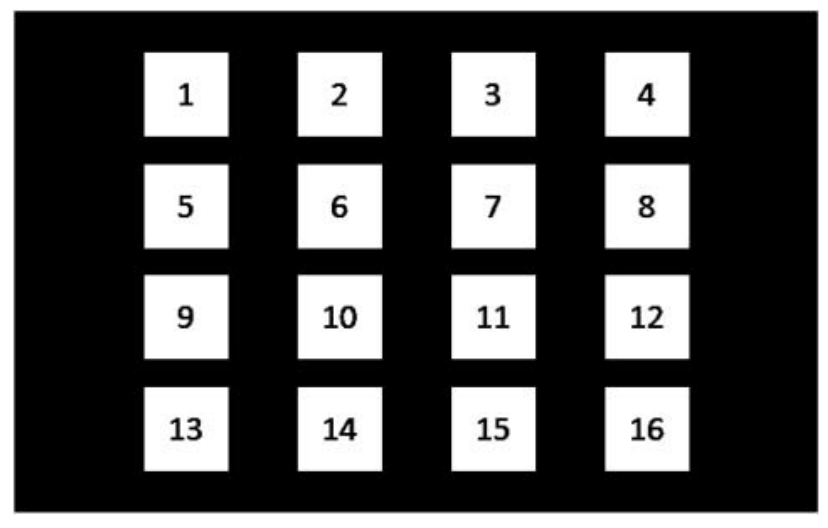

Figure 4. The 16 Flickers on LCD

16 stimuli were 150 pixels x 150 pixels arranged in a $4 \times 4$ matrix.

Participants gazed to the flicker which had frequency of $15 \mathrm{~Hz}$. During the experiment, participant gazed to flicker 6 seconds, after that 4 seconds for resting time. 10 trials were examined by each participants for every duty cycle ratio and signal types that include type I and type II signals.

\subsection{Data Analysis}

Canonical correlation analysis (CCA) is a multivariate analysis that presented by Lin[16]. According to the Equation 2 CCA investigates the maximum correlation between the two variables.

$$
\begin{aligned}
& \max \rho(x, y)=\frac{E\left[x^{T} y\right]}{\sqrt{E\left[x^{T} x\right] E\left[y^{T} y\right]}} \\
& =\frac{E\left[W_{X}^{T} X Y^{T} W_{Y}\right]}{\sqrt{E\left[W_{X}^{T} X X^{T} W_{X}\right] E\left[W_{Y}^{T} Y Y^{T} W_{Y}\right]}}
\end{aligned}
$$

The weight vectors $W_{x}$ and $W_{y}$ to maximize the correlation between, linear combinations, $\mathrm{x}$ and $\mathrm{y}$ by Equation 2 . 
$\max \rho(x, y)$ denoted as the maximum canonical correlation of the $W_{x}$ and $W_{y}$. As shown in Figure 5, shows the CCA model was presented. According to the model, $\mathrm{X}$ denoted EEG signal, $Y_{f}$ denoted the reference signal.

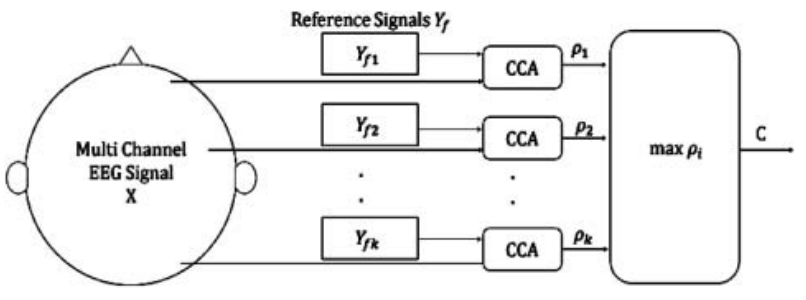

Figure 5. The CCA Model for SSVEP response analysis.

The reference signals were artificially generated with sinus and cosines waves of the flicker's frequency and their harmonics by Equation 3.

$Y_{f}=\left(\begin{array}{c}\sin (2 \pi f t) \\ \cos (2 \pi f t) \\ \cdot \\ \cdot \\ \sin (2 \pi H f t) \\ \cos (2 \pi H t)\end{array}\right), t=\frac{1}{F_{s}}, \frac{2}{F_{s}}, \ldots, \frac{N_{t}}{F_{s}}$

Sixth-order Butterworth band pass filter with 5 and $20 \mathrm{~Hz}$ cut-off frequencies was applied. After that CCA method was used.

$\mathrm{C} W_{x}$ showed that brain frequency map. According to the result, $O_{z}, O_{1}, O_{2}$ EEG channels were used for data accuracy.

ITR is a very significant unit that has been used to evaluate overall performance of BCI systems. ITR can be described by following Equation.

$\frac{\text { Bits }}{\text { Command }}=\log _{2} N+P \log _{2} P+(1-P) \log _{2}\left[\frac{(1-P)}{(N-1)}\right]$

$I T R=\frac{\text { Bits }}{\text { Command }} \times \frac{60}{C T I}$

Where $\mathrm{P}$ denotes accuracy of classification, $\mathrm{N}$ denotes total stimuli, CTI denotes command transfer intervals.

In this study, CTI is 6 seconds because of gaze time during in one trial.

\section{Result and Discussion}

Experiments were completed without any problem. Table 2 shows that outcome of the experiment average accuracy and the average ITR.

Table 1. Result of experiment according to the CCA based method.

\begin{tabular}{|c|c|c|c|}
\hline $\begin{array}{c}\text { Duty } \\
\text { Cycle } \\
\text { Ratio }\end{array}$ & $\begin{array}{c}\text { Signal } \\
\text { Type }\end{array}$ & $\begin{array}{c}\text { Average } \\
\text { Accuracy } \\
(\%)\end{array}$ & $\begin{array}{c}\text { Average } \\
\text { ITR (bpm) }\end{array}$ \\
\hline $40 \%$ & Type I & 88.03 & 30.04 \\
\hline $40 \%$ & Type II & 92.54 & 32.89 \\
\hline $50 \%$ & Type I & 84.98 & 28.32 \\
\hline $50 \%$ & Type II & 90.08 & 31.42 \\
\hline $60 \%$ & Type I & 80.68 & 25.24 \\
\hline $60 \%$ & Type II & 83.28 & 26.92 \\
\hline
\end{tabular}

84 | IJAMEC, 2016, 4(Special Issue), 82-85
Average ITR was improved $16.1 \%$ according to the most commonly used flicker model which is square wave and has $50 \%$ duty cycle. As shown in Figure 6, the highest average accuracy was reached when the flicker's duty cycle ratio was $40 \%$ and type II signal.

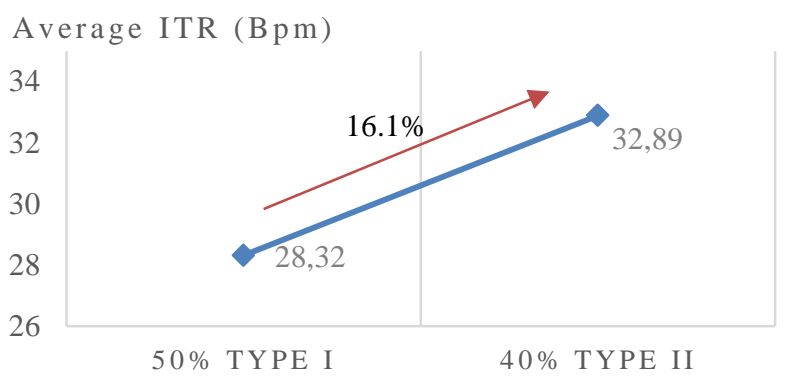

Figure 6. The difference of average ITR and improved ratio.

As written before, ITR is a very important metric to show the overall performance of BCI systems. There are different methods to find out a high ITR; (1) increasing total stimuli, (2) improving the accuracy, and (3) decreasing the command transfer intervals. In this study, we aimed to improve ITR value by increasing the accuracy with a new created signal type for stimuli.

We applied statistical test to the results shown in Table 2 to examine whether the differences in the classification accuracy were significant depending on the duty cycle and flicker type. A one-way analysis of variance (ANOVA) was conducted on the classification of accuracy for each flicker models, indicating that the difference was considered significant at the p-value $<0.05$.

\section{Conclusion}

It can be concluded that previous studies mainly focused on the effects of either duty cycle pulses ratio or brightness of stimuli separately. But, we aimed to investigate the effects of both parameters at the same time. We optimized signal type and duty cycle ratio.

Despite the fact that there is not enough knowledge for characteristic attitude of brightness and duty cycle parameters which are very important for ITR and accuracy in SSVEP-based BCI systems, in this study, for these parameters remarkable results have been accomplished.

\section{References}

[1] Weiskopf N., Mathiak K., Bock S.W., Scharnowski F., Veit R., Grodd W., Goebel R., and Birbaumer N., "Principles of a brain-computer interface (BCI) based on real-time functionalmagnetic resonance imaging (fMRI)," IEEE Trans. Biomed. Eng., vol. 51, no. 6, , Jun. 2004, pp. 966 970.

[2] Sitaram R., Caria A., and Birbaumer N., "Hemodynamic brain-computer interfaces for communication and rehabilitation," Neural Netw., vol. 22, Nov. 2009, pp. 1320-1328.

[3] Muller-Putz G. R. and Pfurtscheller G., "Control of an electrical prosthesis with an SSVEP-based BCI," IEEE Trans. Biomed. Eng., vol. 55, no. 1, Jan. 2008, pp. 361364. 
[4] Donchin E., Spencer K. V., and Wijesinghe R., "The mental prosthesis: Assessing the speed of a P300-based braincomputer interface," IEEE Trans. Rehab. Eng., vol. 8(2), Jun. 2000, pp. 174-179.

[5] Birbaumer N., Kubler A., Ghanayim N., Hinterberger T., Perelmouter J., Kaiser J., Iversen I., Kotchoubey B., Neumann N., and Flor H., "The thought translation device (TTD) for completely paralyzed patients," IEEE Trans. Rehab. Eng., vol. 8(2), , Jun. 2000, pp. 190-193.

[6] Pfurtscheller G., and Neuper C., "Motor imagery and direct brain computer communication," Proc. IEEE, vol. 89(7), Jul. 2001.pp. 1123-1134.

[7] Zhang Y., Zhou G., Jin J., Wang X., and Cichocki A., "Optimizing spatial patterns with sparse filter bands for motor-imagery based brain-computer interface," J. Neurosci. Methods, vol. 255, Nov. 2015, pp. 85-91.

[8] Wang Y., Wang R., Gao X., Hong B., and Gao S., "A practical VEP-based brain-computer interface," IEEE Transactions on Neural Systems and Rehabilitation Engineering, vol. 14, 2006, pp. 234-240.

[9] Wang Y., Wang Y. T., and. Jung T. P, "Visual stimulus design for high-rate SSVEP BCI," Electron Lett., vol. 46 (15) , Jul. 2010, pp. 1057-1058.

[10] Oralhan Z. and Tokmakçı M.. "The Effect of Duty Cycle and Brightness Variation of Visual Stimuli on SSVEP in Brain Computer Interface Systems." IETE Journal of Research (doi: 10.1080/03772063.2016.1176543), 1-9, 2016.

[11] Dal Seno B., Matteucci M., and Mainardi L.T., "The utility metric: A novel method to assess the overall performance of discrete braincomputer interfaces," IEEE Trans. Neural Syst. Rehabil. Eng., vol. 18(1), Feb. 2010, pp. 2028.

[12] Wu Z., "The difference of SSVEP resulted by different pulse duty-cycle," in Communications, Circuits and Systems ICCCAS 2009 International Conference, Milpitas, CA, Jul. 2009, pp. 605-607.

[13] Shyu K. K., Chiu Y. J., Lee P. L., Liang J.M., and Peng S. H., "Adaptive SSVEP-based BCI system with frequency and pulse duty-cycle stimuli tuning design," IEEE Trans. Neural Syst. Rehabil. Eng., vol. 21(5), 2013 pp. 697-703.

[14] Manyakov N. V., Chumerin N., Robben A., Combaz A., Van Vliet M., and Van Hulle M. M., "Sampled sinusoidal stimulation profile and multichannel fuzzy logic classification for monitor-based phase-coded SSVEP braincomputer interfacing," J. Neural Eng., vol. 10(3), Apr. 2013, pp. 115.

[15] Chen X., Chen Z., Gao S., and Gao X., "A high-ITR SSVEP-based BCI speller," Taylor Francis Brain-Comput. Interfaces, vol. 1(34), Sept. 2014, pp. 181-191.

[16] Lin Z., Zhang C., Wu W., and Gao X., "Frequency recognition based on canonical correlation analysis for SSVEPbased BCIs," IEEE Trans. Biomed. Eng., vol. 53(12), Jun. 2007, pp.2610-2614. 